\title{
artigo
}

\section{Qualidade de vida e bem-estar subjetivo de idosos no programa de academia de saúde}

Quality of life and subjective well-being of the elderly in the health academy program

Calidad de vida y bienestar subjetivo de ancianos en el programa academia de la salud

\section{RESUMO}

Objetivo: levantar o perfil sociodemográfico e as condições de qualidade de vida e bem-estar subjetivo de idosos participantes de academias de saúde investigando as correlações significativas entre esses dados. Método: Trata-se de um estudo transversal desenvolvido no município de Sinop, Mato Grosso, com 82 idosos, no mês de janeiro de 2019, sendo utilizados os seguintes instrumentos: Questionário Sociodemográfico, WHOQOL-bref, e as escalas: Satisfação com a vida, Bem-Estar de Afetos Positivos e Negativos e Felicidade Subjetiva. Resultados: Quanto melhor a QV no domínio físico, melhor a QV nos domínios psicológico, relações sociais, meio ambiente, percepção da QV geral, satisfação com a saúde e ao BES relacionado à satisfação com a vida, aos afetos positivos e à felicidade, ainda que não se possa afirmar uma causalidade nessas relações. Conclusão: A Academia de Saúde é de suma importância na contribuição de um envelhecimento saudável da população tornando-se uma estratégica para ações de prevenção e promoção de políticas públicas voltadas a população idosa.

DESCRITORES: Qualidade de vida; Atividade motora; Saúde do Idoso; Psicologia.

\section{ABSTRACT}

Objective: to survey the sociodemographic profile and the conditions of quality of life and subjective well-being of elderly participants in health academies, investigating the significant correlations between these data. Method: This is a cross-sectional study carried out in the municipality of Sinop, Mato Grosso, with 82 elderly people, in January 2019, using the following instruments: Sociodemographic Questionnaire, WHOQOL-bref, and the scales: Life satisfaction, Well-Being of Positive and Negative Affections and Subjective Happiness. Results: The better the QOL in the physical domain, the better the QOL in the psychological, social relations, environment, perception of general QOL, satisfaction with health and BES related to life satisfaction, positive affections and happiness, even that causality cannot be affirmed in these relationships. Conclusion: The Health Academy is of paramount importance in contributing to a healthy aging of the population, becoming a strategy for prevention and promotion of public policies aimed at the elderly population.

DESCRIPTORS: Quality of life; Motor activity; Health of the Elderly; Psychology.

\section{RESUMEN}

Objetivo: relevar el perfil sociodemográfico y las condiciones de calidad de vida y bienestar subjetivo de los ancianos participantes en las academias de salud, investigando las correlaciones significativas entre estos datos. Método: Se trata de un estudio transversal realizado en el municipio de Sinop, Mato Grosso, con 82 ancianos, en enero de 2019, utilizando los siguientes instrumentos: Cuestionario Sociodemográfico, WHOQOL-bref, y las escalas: Satisfacción con la vida. , Bienestar de los Afectos Positivos y Negativos y Felicidad Subjetiva. Resultados: Cuanto mejor es la CV en el dominio físico, mejor es la CV en el ámbito psicológico, relaciones sociales, medio ambiente, percepción de CV general, satisfacción con la salud y BES relacionados con la satisfacción con la vida, afectos positivos y felicidad, incluso que la causalidad no se puede afirmar en estas relaciones. Conclusión: La Academia de la Salud es de suma importancia para contribuir a un envejecimiento saludable de la población, convirtiéndose en una estrategia de prevención y promoción de políticas públicas dirigidas a la población anciana.

DESCRIPTORES: Calidad de vida; Actividad del motor; Salud de los ancianos; Psicología.

RECEBIDO EM: 10/12/2020 APROVADO EM: 17/12/2020

\section{Marieli Basso Bolpato}

Enfermeira e Fisioterapeuta. Doutoranda em Psicologia, Professora na Universidade Federal de Mato Grosso, MT, Brasil.

ORCID: 0000-0002-7923-2343 


\section{Sebastião Benício da Costa Neto}

Psicólogo. Doutor em Psicologia pela Universidade de Brasília (UnB). Pós-Doutor pela Universidade Federal do Rio Grande do Sul (2007) e pela Universidade do Minho (2018).

ORCID: 0000-0001-8160-3476

\section{Ivone Félix Sousa}

Psicóloga: Mestre em Psicologia, Professora na Pontifícia Universidade Católica de Goiás, Goiânia, Brasil. ORCID: 0000-0001-9231-9534

\section{INTRODUÇÃO}

A Qualidade de Vida (QV) e o bem-estar subjetivo (BES) são preocupações constantes na vida dos indivíduos, consistindo-se em temas de interesse da literatura especializada desde o século passado e possibilitando a ampliação da busca pela melhor compreensão da realidade de diversos seguimentos sociais, a exemplo da população de idosos brasileiros ${ }^{1}$.

A pessoa idosa apresenta fatores indicativos de QV e BES, sendo que tais fatores são intrínsecos (personalidade, espiritualidade, interpretações subjetivas de eventos, dentre outros) e extrínsecos (condições de saúde, renda, educação, situação conjugal, apoio social, dentre outros ${ }^{2}$. Assim, o envelhecimento é um fenômeno que consiste em um processo natural e progressivo que ocorre em todas as espécies. No ser humano, particularmente, este processo é verificado nas dimensões físicas, mentais e sociais que, não ocorre de forma simultânea necessariamente, mas, podem interferir acelerando ou diminuindo o processo de envelhecimento ${ }^{3}$.

O conceito de QV tem sido considerado como um fenômeno multidimensional e complexo, envolvendo a percepção subjetiva de cada pessoa acerca de aspectos objetivos, subjetivos e de interfaces entre esses dois ${ }^{4,5}$. Já o conceito BES é uma reestruturação do conceito de bem-estar geral na medida em que compreende a subjetividade dos aspectos positivos e negativos, a felicidade e a satisfação com a vida ${ }^{67}$.

Neste sentido, o Programa de Academia de Saúde é direcionado a atender estratégias de Promoção a Saúde por meio da implantação de Academia de Saúde (AS) e foi criado no Brasil, em 2011, com a implementação de ações voltadas às práticas corporais em grupo destacando- -se como uma ferramenta qualificada da Atenção Básica de saúde com o propósito de fortalecimento das ações preventivas na comunidade ${ }^{8,9}$.

$A Q V$ e o $B E S$ são variáveis importantes a serem consideradas em estudos gerontológicos, pois possibilitam avaliar o comportamento dos idosos em relação ao grau de adaptação diante as modificações físicas, psicológicas e suas relações sociais no processo de envelhecer ${ }^{10,11}$.

Diante do exposto e considerando-se a falta de evidências sobre as condições de $\mathrm{QV}$ e de BES dos idosos em AS, este estudo teve como objetivo levantar o perfil sociodemográfico e as condições de QV e de BES dos idosos que participam do programa AS, assim como, investigar as correlações significativas entre os dados sociodemográficos, as dimensões de QV e de BES (a satisfação com a vida, os afetos positivos e negativos e a felicidade) de idosos frequentadores de AS.

\section{MÉTODO}

Estudo de abordagem quantitativa, transversal e correlacional que se desenvolveu no município de Sinop, Mato Grosso. Constituíram a amostra 82 idosos, entre 60 e 81 anos de idade, de ambos os sexos, que participavam ativamente do Programa de Academia de Saúde. Foram incluídos idosos com 60 anos ou mais cadastrados na AS com assiduidade de pelo menos uma vez na semana. Foram excluídos aqueles que deixaram de responder a algum item dos instrumentos de investigação.

A coleta de dados foi realizada na AS, no mês de Janeiro (2019), por meio de aplicação individual dos instrumentos. Os dados sociodemográficos e a avaliação dos idosos referentes a satisfação com AS foram obtidos por meio de formulário estruturado em três eixos: (1) dados sociodemográficos (idade, sexo, estado civil, religião, grau de religiosidade, grau de escolaridade, número de pessoas em sua moradia e dependentes); (2) frequencia dos idosos à AS (meses de participação, frequência semanal e atividades realizadas); e, (3) importância/relevância da AS para os idosos e o grau de satisfação e motivação.

Para avaliar a QV foi utilizado o questionário Whoqol-bref considerado uma escala tipo Likert composta por 26 frases, distribuídas em quatro domínios (físicos, psicológicos, relações sociais e meio ambiente) e duas perguntas ( 1 e 2) que se referem a QV geral, em que o item 1 avalia a percepção da $Q V$ e o 2 avalia a satisfação com a saúde. As respostas seguem a escala Likert e varia de 1 a 5 , em que quanto maior for a pontuação, melhor é a $\mathrm{QV}^{8}$.

Para avaliar o BES utilizou-se da escala de satisfação com a vida - SLWS elaborada por Diener', e tem por objetivo avaliar a satisfação com a vida ${ }^{12}$. Assim, quanto maior a pontuação obtida no resultado final, melhor é a satisfação com a vida do respondente ${ }^{10,11}$. Outro instrumento utilizado foi a Escala PANAS desenvolvida e validada por Watson, Clark e Tellegen ${ }^{12}$ com o objetivo de mensurar a experiência afetiva dos indivíduos a partir das dimensões: Afeto Positivo (AP) e Afeto Negativo $(\mathrm{AN})^{13}$. Na dimensão $\mathrm{AN}$ são avaliados o desprazer e o mal-estar subjetivo e inclui emoções como o medo, o nervosismo e a perturbação. Na dimensão dos AP, avalia-se o prazer e o bem-estar subjetivo, sendo que neste estão inclusas as emoções de entusiasmo, inspiração e determinaçã̃o ${ }^{14}$.

Por fim, utilizou-se a escala de felicidade subjetiva - SHS, construída e validada por Lyubomirsky e Lepper ${ }^{15}$ com o objetivo de avaliar a felicidade na perspectiva do respondente, a partir de seu julgamento 


\section{artigo}

Bolpato, M.B.; Costa-Neto, S.B.; Sousa, I.F.

Qualidade de vida e bem-estar subjetivo de idosos no programa de academia de saúde

geral, no quanto se sente feliz, independente de suas vivências recentes ${ }^{16}$.

O projeto de pesquisa foi aprovado no Comitê de Ética de Pesquisa da PUG Goiás sobre o CAAE: 89996318.5.0000.0037 e, respeitando a Resolução no 466, de 12 de dezembro de 2012 que dispõe de diretrizes e normas regulamentadoras de pesquisas envolvendo seres humanos. $\mathrm{Na}$ sequência, os idosos da AS foram abordados pessoalmente e convidados a participar do estudo. Com os mesmos, foi realizada a leitura e a coleta de assinatura no Termo de Consentimento Livre e Esclarecido (TCLE).

Para as análises de dados foi utilizado o pacote de software Statistical Package for Social Sciences (SPSS) versão 22.0. Foram realizadas análises descritivas para os dados sociodemográficos, níveis de QV e de BES. Utilizou-se também a análise correlacional para as variáveis sociodemográficas, QV e BES dos sujeitos pesquisados.

Conforme o The Whoqol Group, $1998^{17}$, os domínios e alfas do instrumento correspondem: Físico $(\alpha=0,829)$; Psicológico $(\alpha=0,793)$; Relações Sociais $(\alpha=0,704)$; Meio Ambiente $(\alpha=0,881)$; e QV Geral $(\alpha=0,877)$. Estes dados indicam que há consistência interna entre os itens e, portanto, a escala é válida para mensurar a QV neste grupo. Considerou-se a normatização para QV os seguintes intervalos (The Whoqol Group, 1998)18 de 1 a 2,9 (necessita melhorar), de 3 a 3,9 (regular), de 4 a 4,9 (boa), e 5 (muito boa).

Para o presente estudo, foi realizada a análise de alfa de Cronbach da escala SLWS10 para confirmar a consistência interna entre os itens e a validade deste instrumento sendo encontrado $\alpha=0,908$, confirmando a validade psicométrica. A análise da escala PANAS possibilitou confirmar a consistência interna dos itens e a validade psicométrica dos afetos positivos (AP) que obteve $\alpha=0,876$ e os afetos negativos (AN) que obteve $\alpha=0,865$. Também foi realizada a análise de alfa de Cronbach para a escala SHS e obteve-se o $\alpha=0,737$, confirmando sua validade psicométrica e apresentando uma consistência melhor do que a de Spagnoli et al. ${ }^{18}$.

\section{RESULTADOS}

Apresentam-se primeiramente os dados sociodemográficos (idade, sexo, estado civil, religião, grau de religiosidade, grau de escolaridade, número de pessoas que moram com os/as idosos/as, e se tem dependentes - Tabela 1). Seguem-se as análises de correlação de Spearman (Tabela 2), onde pode-se verificar as relações entre os dados sociodemográficos, os domínios da $\mathrm{QV}$ e o BES em relação à satisfação com a vida (SLWS), aos afetos positivos (AP) e negativos (AN) e à felicidade (SHS). Levou-se em consideração as relações estatísticas significativas para $\mathrm{p} \leq 0,05$.

$\mathrm{Na}$ Tabela 1, 82 idosos responderam ao presente estudo e destes, houve prevalência da faixa etária de 60 a 70 anos (70,7\%,), do gênero feminino $(70,7 \%)$, casados $(56,1 \%)$, católicos $(68,3 \%)$, com ensino médio completo $(32,9 \%)$ (Tabela 1$)$.

A Tabela 2, na análise de correlação, em relação à idade, observou-se que há correlação negativa significativa $(\mathrm{r}=$ $-0,209 ; \mathrm{p}=0,001)$ entre o número de pessoas que residem, o domínio físico $(\mathrm{r}=$ $-0,313 ; \mathrm{p}=0,000)$, o domínio psicológico $(\mathrm{r}=-0,201 ; \mathrm{p}=0,002)$, as relações sociais $(r=-0,348 ; p=0,000)$, o meio ambiente $(r=-0,196 ; p=0,002)$, a percepção da qualidade de vida geral $(\mathrm{r}=-0,148$; $\mathrm{p}=0,023)$, e a satisfação com a saúde $(\mathrm{r}=$ $-0,133 ; \mathrm{p}=0,041)$. Assim, pode-se inferir que, quanto maior a idade dos idosos, menor o número de pessoas que residem com os mesmos e piores estão em relação aos domínios físico e psicológico, relações sociais, meio ambiente, percepção da qualidade de vida (QV) e a satisfação com a saúde Geral. No entanto, não houve correlação significativa entre a idade e as variáveis de BES (SLWS, AP, AN e SHS) (Tabela 2).

Em relação aos resultados obtidos com a variável tempo de academia notou-se a existência de correlações positivas significativas entre domínios físico $(\mathrm{r}=$ $0,165 ; \mathrm{p}=0,011)$, psicológico $(\mathrm{r}=0,202$; $\mathrm{p}=0,002)$, percepção da $\mathrm{QV}$ geral $(r=0,272 ; p=0,000)$, satisfação com a saúde geral $(\mathrm{r}=0,171 ; \mathrm{p}=0,008)$, assim como com o BES em relação a SLWS $(r=0,272$; $\mathrm{p}=0,000)$, e a SHS $(\mathrm{r}=0,343 ; \mathrm{p}=0,000)$. Estes dados possibilitam inferir que quanto maior o tempo de academia, melhor é a QV nos domínios físico, psicológico, na percepção da QV geral e na satisfação com a saúde, assim como, em relação ao $\mathrm{BES}$ relativos à satisfação com a vida e à felicidade (Tabela 2).

Ao correlacionar domínio físico da QV

\section{Tabela 1: Descrição sociodemográfica dos idosos participantes da AS no} município de Sinop-MT (N=82).

\begin{tabular}{lccc} 
Variáveis & Categorias & N & $\%$ \\
& 60 a 70 & 58 & 70,7 \\
Idade & 71 a 80 & 22 & 26,8 \\
& $>80$ & 2 & 2,4 \\
\multirow{2}{*}{ Gênero } & Masculino & 24 & 29,3 \\
& Feminino & 58 & 70,7 \\
Estado Civil & Solteiro(a), viúvo(a), separado(a) e sem parceiro(a) regular & 36 & 43,9 \\
& Casado(a), união estável, com parceiro(a) regular & 46 & 56,1 \\
Religião & Católico(a) & 56 & 68,3 \\
& Ensino Fundamental Completo & 15 & 18,3 \\
Escolaridade & Ensino Fundamental Incompleto & 13 & 15,9 \\
& Ensino Médio Completo & 27 & 32,9 \\
Total & & 82 & 100 \\
Fonte: Elaborado pela autora (2019), Sinop-MT. & &
\end{tabular}


com as demais variáveis de QV e BES, observou que existem correlações significativas positivas entre os domínios da $\mathrm{QV}$ : psicológico $(r=0,609 ; \mathrm{p}=0,000)$, relações sociais $(\mathrm{r}=0,634 ; \mathrm{p}=0,000)$, meio ambiente $(\mathrm{r}=0,632 ; \mathrm{p}=0,000)$, percepção da $\mathrm{QV}$ geral $(r=0,418 ; p=0,000)$ e satisfação geral com saúde ( $\mathrm{r}=0,353 ; \mathrm{p}=0,000)$; $\mathrm{e}$ as dimensões que mensuram o $\mathrm{BES}$ : satisfação com a vida $(r=0,424 ; p=0,000)$, afetos positivos $(\mathrm{r}=0,239 ; \mathrm{p}=0,000)$ e felicidade $(\mathrm{r}=0,276$; $\mathrm{p}=0,000)$. Com estes dados, pode-se dizer que quanto melhor a $\mathrm{QV}$ no domínio físico, melhor a QV nos domínios psicológico, relações sociais, meio ambiente, percepção da QV geral, satisfação com a saúde e ao $\mathrm{BES}$ relacionado à satisfação com a vida, aos afetos positivos e à felicidade, ainda que não se possa afirmar uma causalidade nessas relações (Tabela 2).

Quando avaliada a $\mathrm{QV}$ no domínio psicológico, notou-se que quanto melhor ele se apresenta, melhor a QV nos domínios: relações sociais $(r=0,535 ; \mathrm{p}=0,000)$, meio ambiente $(r=0,556 ; p=0,000)$, percepção da QV geral $(r=0,432 ; p=0,000)$, satisfação com a saúde $(r=0,406$; $\mathrm{p}=0,000)$; e o $\mathrm{BES}$ : satisfação com a vida $(r=0,588 ; p=0,000)$, afetos positivos $(r=$ $0,478 ; \mathrm{p}=0,000)$ e felicidade $(\mathrm{r}=0,399$; $\mathrm{p}=0,000)$. Ao avaliar o domínio das relações sociais verificou-se correlação estatística significativa de forma positiva nos domínios da $\mathrm{QV}$ : meio ambiente $(\mathrm{r}=0,561$; $\mathrm{p}=0,000)$, percepção $\mathrm{da} \mathrm{QV}$ geral $(\mathrm{r}=$ 0,205; $p=0,001)$, satisfação com a saúde $(\mathrm{r}=0,188 ; \mathrm{p}=0,000)$; e o BES: satisfação com a vida $(r=0,354 ; p=0,000)$, afetos positivos $(\mathrm{r}=0,227 ; \mathrm{p}=0,000)$ e felicidade $(\mathrm{r}=0,209 ; \mathrm{p}=0,001)$ (Tabela 2).

Observou-se que há correlação positiva significativa entre o domínio da $\mathrm{QV}$ meio ambiente e os domínios da QV: percepção da QV geral ( $r=0,282 ; p=0,000)$, a satisfação com a saúde $(r=0,181 ; p=0,005)$; e o BES: a satisfação com a vida $(r=0,419$; $\mathrm{p}=0,000)$, afetos positivos $(\mathrm{r}=0,229$; $\mathrm{p}=0,000)$ e felicidade $(\mathrm{r}=0,185 ; \mathrm{p}=0,000)$. Estes dados possibilitam inferir que quanto melhor o meio ambiente, melhor percebida a $Q V$, a satisfação com a saúde e a vida, os afetos positivos e a felicidade.

Já em relação à percepção da $\mathrm{QV}$ observou-se que há correlação estatística po- sitiva significativa com a satisfação com a saúde $(\mathrm{r}=0,598 ; \mathrm{p}=0,000)$ e com o BES relacionado à satisfação com a vida ( $\mathrm{r}=$ $0,412 ; \mathrm{p}=0,000)$, aos afetos positivos $(\mathrm{r}=$ $0,247 ; \mathrm{p}=0,000)$ e à felicidade $(\mathrm{r}=0,365$; $\mathrm{p}=0,000)$. A QV no domínio satisfação com a saúde obteve correlação estatística significativa com o BES em relação à satisfação com a vida ( $r=0,351 ; p=0,000)$, aos afetos positivos $(\mathrm{r}=0,257 ; \mathrm{p}=0,000)$ e a felicidade $(\mathrm{r}=0,356 ; \mathrm{p}=0,000)$ (Tabela 2).

Ao avaliar o BES relativo à satisfação com a vida observou-se que houve correlação estatística positiva significativa com o BES relacionado aos afetos positivos $(\mathrm{r}=0,430 ; \mathrm{p}=0,000)$ e à felicidade $(\mathrm{r}=0,419 ; \mathrm{p}=0,000)$, possibilitando inferir que quanto melhor a satisfação com a vida, mais afetos positivos e melhor a felicidade dos idosos. Contudo, o BES avaliado a partir da satisfação com a vida mostrou correlação estatística significativa negativa com os afetos negativos $(r=-0,168 ; p=0,000)$ evidenciando que, quanto menor a satisfação com a vida, mais afetos negativos são apresentados pelas pessoas idosas (Tabela 2).

\section{Tabela 2 - Correlações entre os dados sociodemográficos, WHOQOL-bref, Escala de Satisfação com a vida-SLWS,} afetos positivos e negativos-PANAS e Escala da felicidade subjetiva (SHS)

\begin{tabular}{|c|c|c|c|c|c|c|c|c|c|c|c|c|c|}
\hline Variáveis & & 1 & 2 & 3 & 4 & 5 & 6 & 7 & 8 & 9 & 10 & 11 & 12 \\
\hline \multirow{2}{*}{ 1. Id } & $r$ & 1 & & & & & & & & & & & \\
\hline & p & & & & & & & & & & & & \\
\hline \multirow{2}{*}{ 2.NPMC } & $r$ & $-0,209^{* *}$ & 1 & & & & & & & & & & \\
\hline & p & 0,001 & & & & & & & & & & & \\
\hline \multirow{2}{*}{ 3.TA } & $r$ & $-0,033$ & $-0,025$ & 1 & & & & & & & & & \\
\hline & $\mathrm{p}$ & 0,610 & 0,697 & & & & & & & & & & \\
\hline \multirow{2}{*}{ 4.DF } & $r$ & $-0,313^{* *}$ & $-0,101$ & $0,165^{*}$ & 1 & & & & & & & & \\
\hline & $\mathrm{p}$ & 0,000 & 0,121 & 0,011 & & & & & & & & & \\
\hline \multirow{2}{*}{ 5.DP } & $r$ & $-0,201^{* *}$ & 0,117 & $0,202^{* *}$ & $0,609^{* *}$ & 1 & & & & & & & \\
\hline & $p$ & 0,002 & 0,071 & 0,002 & 0,000 & & & & & & & & \\
\hline \multirow{2}{*}{ 6.DRS } & $r$ & $-0,348^{* *}$ & 0,072 & 0,077 & $0,634^{* *}$ & $0,535^{* *}$ & 1 & & & & & & \\
\hline & $\mathrm{p}$ & 0,000 & 0,266 & 0,240 & 0,000 & 0,000 & & & & & & & \\
\hline \multirow{2}{*}{ 7.DMA } & $r$ & $-0,196^{* *}$ & 0,091 & 0,050 & $0,632^{* *}$ & $0,556^{* *}$ & $0,561^{\text {** }}$ & 1 & & & & & \\
\hline & $p$ & 0,002 & 0,162 & 0,439 & 0,000 & 0,000 & 0,000 & & & & & & \\
\hline \multirow{2}{*}{ 8.PQVg } & $r$ & $-0,148^{*}$ & 0,059 & $0,272^{* *}$ & $0,418^{* *}$ & $0,432^{* *}$ & $0,205^{* *}$ & $0,282^{* *}$ & 1 & & & & \\
\hline & $p$ & 0,023 & 0,362 & 0,000 & 0,000 & 0,000 & 0,001 & 0,000 & & & & & \\
\hline
\end{tabular}




\begin{tabular}{|c|c|c|c|c|c|c|c|c|c|c|c|c|c|}
\hline \multirow{2}{*}{ 9.SS } & $r$ & $-0,133^{*}$ & 0,068 & $0,171^{* *}$ & $0,353^{* *}$ & $0,406^{* *}$ & $0,188^{* *}$ & $0,181^{* *}$ & $0,598^{* *}$ & 1 & & & \\
\hline & $p$ & 0,041 & 0,293 & 0,008 & 0,000 & 0,000 & 0,004 & 0,005 & 0,000 & & & & \\
\hline \multirow{2}{*}{ 10.SLWS } & $r$ & $-0,088$ & 0,109 & $0,272^{* *}$ & $0,424^{* *}$ & $0,588^{* *}$ & $0,354^{* *}$ & $0,419^{* *}$ & $0,412^{* *}$ &, $351^{* *}$ & 1 & & \\
\hline & $p$ & 0,177 & 0,094 & 0,000 & 0,000 & 0,000 & 0,000 & 0,000 & 0,000 & 0,000 & & & \\
\hline \multirow{2}{*}{ 11.AP } & $r$ & $-0,031$ & $-0,005$ & 0,123 & $0,239 * *$ & $0,478^{* *}$ & $0,227^{* *}$ & $0,229^{* *}$ & $0,247^{* *}$ & $0,257^{* *}$ & $0,430^{* *}$ & 1 & \\
\hline & $\mathrm{p}$ & 0,632 & 0,943 & 0,059 & 0,000 & 0,000 & 0,000 & 0,000 & 0,000 & 0,000 & 0,000 & & \\
\hline \multirow{2}{*}{ 12.AN } & r & 0,018 & 0,063 & 0,084 & $-0,007$ & $-0,116$ & $-0,034$ & $-0,062$ & $-0,013$ & $-0,060$ & $-0,168^{* *}$ & $-0,448^{* *}$ & 1 \\
\hline & $p$ & 0,778 & 0,335 & 0,199 & 0,920 & 0,074 & 0,607 & 0,345 & 0,839 & 0,355 & 0,009 & 0,000 & \\
\hline \multirow{2}{*}{ 13.SHS } & $r$ & $-0,007$ & 0,098 & $0,343^{* *}$ & $0,276^{* *}$ & $0,399^{* *}$ & $0,209^{* *}$ & $0,185^{* *}$ & $0,365^{* *}$ & $0,356^{* *}$ & $0,419^{* *}$ & $0,382^{* *}$ & $-0,045$ \\
\hline & p & 0,917 & 0,132 & 0,000 & 0,000 & 0,000 & 0,001 & 0,000 & 0,000 & 0,000 & 0,000 & 0,000 & 0,490 \\
\hline
\end{tabular}

Por fim, em relação ao BES relativo aos afetos positivos observou-se correlação estatística negativa significativa com os afetos negativos $(r=-0,448 ; p=0,000)$ e positiva com a felicidade $(r=0,382 ; \mathrm{p}=0,000)$. Estes dados possibilitam inferir que quanto mais afetos negativos são vivenciados pelas pessoas idosas, menos afetos positivos elas apresentam e que quanto mais afetos positivos vivenciados, mais felicidade os idosos percebem (Tabela 2).

\section{DISCUSSÃO}

As academias de saúde proporcionam à pessoa idosa o estabelecimento de redes de apoio social que pode contribuir com a melhora da QV e do BES. Os dados demonstraram que a AS apresenta-se como relevante para saúde em geral, vida pessoal, fazer amizades, bem-estar psicológico geral, interações sociais, diminuição de desafetos sociais e familiares e para vivenciar o processo de envelhecimento, corroborando com os autores ${ }^{19}$ que apontaram que aspectos saudáveis do desenvolvimento humano perpassam por relações sociais que envolvam os vínculos afetivos familiares e se estendem para os laços afetivos de amizade entre pares (membros da comunidade) construídos durante todo o ciclo vital.

A importância da AS no fortalecimento dos vínculos familiares e sociais (amizades) vai ao encontro de autores ${ }^{20}$ que demonstraram a manutenção do vínculo familiar afetivo fortalecendo os afetos positivos e a satisfação com a vida. Desta forma, os autores apontam que a idade avançada e a redução das capacidades funcionais (cognitivas e afetivas) requer suporte familiar para uma melhor percepção da QV e BES nessa população ${ }^{21}$.

\section{A importância \\ da AS no \\ fortalecimento dos vínculos familiares}

\section{e sociais...}

Em suma, os idosos mostraram melhora na percepção da $\mathrm{QV}$ e BES oriundas das práticas de atividades físicas como dança, exercícios e/ou alongamentos, verificando que a $\mathrm{QV}$ no domínio físico obteve correlações significativas positivas com a $\mathrm{QV}$ psicológica, com as relações sociais, com a percepção da QV geral, com a satisfação com saúde e a vida, com os afetos positivos e com a felicidade ${ }^{19}$.

$\mathrm{O}$ aumento do interesse da pessoa idosa pela procura de práticas físicas está correlacionado ao fato de pertencer a grupos de idosos(as) ativos(as), independentes e motivados(as) pela $\mathrm{AS}^{16}$. Isto vai ao encontro de autores que verificaram a importância da manutenção do domínio físico oriundos de práticas aeróbicas de baixa intensidade em idosos entre $60 \mathrm{e}$ 70 anos, possibilitando evidenciar o aumento significativo e positivo pela busca de programas voltados a prevenção no surgimento de morbidades, a fim de obter melhora com a satisfação com a vida, com o condicionamento físico, psicológico, relações sociais e $\mathrm{QV}^{22}$.

Percebeu-se correlação significativa negativa entre os afetos positivos e negativos compreendendo que, quanto mais alto os níveis de desafetos na vida dos(as) ido$\operatorname{sos}(a s)$ (medo, raiva), pior a percepção de afetos positivos (felicidade). Estes dados leva a reflexão de como é importante o apoio psicológico nas intervenções individuais em relação aos eventos negativos desfavoráveis (afetos negativos) do processo de envelhecimento, principalmente no que se refere às reduções das capacidades e aceitação das limitações funcionais cognitivas ${ }^{21}$.

Neste contexto, observou-se que as relações sociais, a percepção da QV geral e a satisfação com a saúde e a vida correlacionaram-se positivamente com os afe- 
tos positivos e negativos e, a felicidade, evidenciando uma compreensão positiva que os(as) idosos(as) fazem em relação à aceitação das limitações do processo de envelhecimento e a melhora na percepção da QV e BES 22 .

Por fim, a felicidade mensurada pelo BES mostrou-se relevante nas percepções de QV e BES nos idosos, reforçando a ne- cessidade de mais pesquisas desenvolvidas sobre a AS, a fim de melhor compreender as vivências e expectativas na longevidade ${ }^{23}$.

\section{CONCLUSÃO}

Concluiu-se que existem correlações significativas em relação ao perfil sociodemográfico, as condições de QV e o BES dos idosos por meio de atividades ofertadas na AS favorecendo, por sua vez, uma melhor percepção da QV e do BES. Os resultados revelam, ainda, a importância de AS na contribuição de um envelhecimento saudável da população o que, no limite, reconhece sua posição estratégica $\mathrm{n}$ nas políticas públicas afins às ações de prevenção e promoção da saúde. •

\section{REFERÊNCIAS}

1. Beauchet O, Bastien T, Mittelman M, Hayashi Y, Ho AHY. Participatory art-based activity, community-dwelling older adults and changes in health condition: Results from a pre-post intervention, single-arm, prospective and longitudinal study. Maturitas. $2020 ; 134: 8-14$

2. Teixeira Indo, Neri AL. Envelhecimento bem-sucedido: uma meta no curso da vida. Rev. psicol USP. 2008; 19(1):81-94.

3. Veras RP, Oliveira M. Envelhecer no Brasil: a construção de um modelo de cuidado. Rev. Ciênc saúde coletiva. 2018; 23(6):192936. 4.

4. Miquilussi PA, de Lima Zanata I, de Andrade Sartori AP, da Silva JTN, da Silva J. A percepção da qualidade de vida do idoso disfágico após intervenção fonoaudiológica. Rev. saúde pública do Paraná. 2019; 2(1):93-102. 5.

5. Costa Neto SB, Araujo T. Qualidade de vida do enfermo oncológico: um panorama sobre o campo e suas formas de avaliação. Temas em psico-oncologia. 2008;195-208.

6. Shafrin J, Sullivan J, Goldman DP, Gill TM. The association between observed mobility and quality of life in the near elderly. PloS one. 2017; 12(8):0182920.

7. Skevington SM, Böhnke JR. How is subjective well-being related to quality of life? Do we need two concepts and both measures? Rev. Social Science e Medicine. 2018; 206:22-30.

8. Domingues JP, Oliveira DC de, Marques SC. Representações sociais da qualidade de vida de pessoas que vivem com HIV/ AIDS. Rev.Texto \& Contexto-Enfermagem. 2018; 27(2).

9. Nunes RP, de Melo RLP, da Silva Júnior EG, do Carmo Eulálio M. Relationship between coping and subjective well-being of elderly from the interior of the Brazilian Northeast. Psicologia: Rev. Reflexão e Crítica. 2016; 29(1):33.

10. Diener E. Subjective well-being: The science of happiness and a proposal for a national index. Acta American psychologist. 2000; 55(1):34.

11. Silva Neto MFD, Soares DJ. Atividade física na terceira idade: um estudo de caso do projeto Universidade Aberta a Terceira Idade (UATI) na cidade de Feira de Santana/BA. Rev. CEFAC. 2016; 18(5): 1088-96.

12. Sá GBAR de, Dornelles GC, Cruz KG, Amorim RC de A, Andrade SSC de A, Oliveira TP, et al. O Programa Academia da Saúde como estratégia de promoção da saúde e modos de vida saudáveis: cenário nacional de implementação. Rev. Ciência \& Saúde Coletiva. $2016 ; 21: 1849-1860$.

13. Jadidi A, Farahaninia $M$, Janmohammadi $S$, Haghani $H$. The Relationship between Spiritual Well-Being and Quality of Life among Elderly People Residing in Kahrizak Senior House. Iran Journal of Nursing (2008-5923). 2011; 24(72).

14. Sinop PM de. Prefeitura Municipal de Sinop. Prefeitura Municipal de Sinop.

15. Spagnoli P, Caetano A, Silva A. Psychometric properties of a Portuguese version of the Subjective Happiness Scale. Rev. Social Indicators Research. 2012; 105(1):137-143.

16. Fleck M, Louzada S, Xavier M, Chachamovich E, Vieira G, Santos $L$, et al. Application of the Portuguese version of the abbreviated instrument of quality life WHOQOL-bref. Rev.Saude Publica. 2000; 34(2):178-183.

17. Canavarro MC, Simões MR, Vaz Serra A, Pereira M, Rijo D, Quartilho MJ, et al. Instrumento de avaliação da qualidade de vida da Organização Mundial de Saúde: WHOQOL-Bref. Avaliação psicológica: Instrumentos validados para a população portuguesa. Rev. Bras. Geriatr. Geront. 2007 [Acesso em 12 de abril. 2020]; 3:77-100.

18. Rech CR, Fermino RC, Hallal PC, Reis RS. Validade e fidedignidade da escala de satisfação com a prática de atividade física em adultos. Rev. Saúde Pública. 2011; 45(2):286-293.

19. Diener ED, Emmons RA, Larsen RJ, Griffin S. The satisfaction with life scale. Journal of personality assessment. 1985; 49(1):71-75.

20. Watson D, Lee AC, Auke T. Development and validation of brief measures of positive and negative affect: the PANAS scales. Journal of personality and social psychology. 1988; 51(6): 56-61.

21. Aghajanian A, Thompson V. Gender and living arrangements of the elderly in Iran. Asian Population Studies. 2016; 12(2):177186.

22. Galinha IC, Pereira CR, Esteves F. Versão reduzida da escala portuguesa de afeto positivo e negativo-PANAS-VRP: Análise fatorial confirmatória e invariância temporal. Cad. psicologia. 2014; 28(1):50-62.

23. Lyubomirsky S, Lepper HS. A measure of subjective happiness: $\mathrm{nPreliminary}$ reliability and construct validation. Social indicators research. 1999; 46(2):137-155. 